\title{
Differentiation between Spinal Intramedullary Astrocytoma and Spinal Multiple Sclerosis Using Clinical and Radiologic Factors
}

\author{
Hyeongyu Jang ${ }^{1}$, Moo Sung Kang ${ }^{2}$ \\ ${ }^{1}$ Department of Neurosurgery, International St. Mary's Hospital, Catholic Kwandong University College of Medicine, Incheon, Republic of Korea \\ ${ }^{2}$ Department of Neurosurgery, Barun Neurosugery Clinic, Goyang, Republic of Korea
}

Corresponding author:

Moo Sung Kang

Department of Neurosurgery,

Barun Neurosugery Clinic, Pastel

City 3F, 893, Gwonyul-daero,

Deogyang-gu, Goyang-si,

Gyeonggi-do, Republic of Korea

Tel: $+82-2-381-7275$

Fax: $+82-2-381-5275$

E-mail: gtrkd@naver.com

Received: March 15, 2021

Revised: August 13, 2021

Accepted: August 18, 2021

\begin{abstract}
Objective: Both spinal multiple sclerosis (SMS) and spinal intramedullary astrocytoma (SIA) are rare space-occupying lesions in the spinal cord and clinically show various myelopathy symptoms. Both clinical and imaging findings are similar and are easily misdiagnosed. This study is to clarify clinical and radiographic finding that could be helpful to differentiate SMS from SIA.

Methods: We compared the demographic, clinical, and radiographic characteristics between the SIA and SMS groups. The SIA group $(n=13)$ was diagnosed postoperatively with pathologic confirmation, and the SMS group $(n=25)$ was composed of patients who have a lesion in the spinal cord and met the McDonald criteria for the diagnosis of multiple sclerosis (criteria revised in 2010).

Results: Clinically, patients with SIA had a significantly longer symptom duration before the first visit than SMS (4.9 \pm 4.4 vs. $2.1 \pm 3.6$ months, $p=0.008)$. All persons with SIA showed progressive disease course, whereas $95.8 \%$ of persons with SMS showed remission $(p<0.001)$. In contrast to SMS, the involvement of both halves of the spinal cord was more frequently observed in patients with SIA $(p<0.001)$. In addition, fusiform dilation in the sagittal plane $(p<0.001)$ or tumoral cyst $(p=0.001)$ also significantly suggested SIA rather than SMS.

Conclusion: Despite many limitations of this study, the present data demonstrated the clinical and imaging features helpful in distinguishing SIA from SMS. As with most tumors, SIA tends to show a slowly progressive clinical course without remission. Occupation of both halves of the spinal cord, fusiform dilation, or cysts was favoring radiographic factor for the SIA.
\end{abstract}

Key Words: Astrocytoma; Diagnosis, differential; Multiple sclerosis; Spinal cord; Spine

\section{INTRODUCTION}

Multiple sclerosis (MS) is the most common demyelinating disease in the central nervous system (CNS). It often involves the spinal cord with $74 \%$ to $95 \%$ in established MS $^{111}$. Although the prevalence of spinal cord abnormality is lower in clinically isolated syndromes, $30 \%$ to $40 \%$ of these patients also had asymptomatic lesions. An acute demyelinating event is offen represented on magnetic resonance imaging (MRI) by solitary or multifocal hyperintensities on T2-weighted images (T2-W) with a variable pattern of enhancement, challenging the distinction of demyelination from intramedullary neoplasms. Differentiation of these two diseases is of great importance in selecting treatment modalities and predicting the patient's prognosis. Although numerous reports have been described the suggestive MRI findings of each disease, a comparative radiological study between these two diseases has rarely been accomplished or has only provided an obscure description. Therefore, in many cases, the differentiation of MS from intramedullary glioma without biopsy has been made without confidence due to similarities in imaging features. This study compares the clinical presentation and radiographic features between
MS and intramedullary astrocytoma in the spinal cord.

\section{MATERIALS AND METHODS}

The Institutional Review Board approved this study. This study is a retrospective design that does not involve an intervention, and the patient's written consent was waived.

\section{Patients}

Between January 2004 and December 2013, spinal intramedullary astrocytoma (SIA) patients were identified based on surgical records and pathologic results in our institutional data set. The spinal MS (SMS) patient group was searched for "multiple sclerosis" as the inpatient and outpatient records diagnosis. The patients who have one or more lesions in the spinal cord and meet the McDonald criteria for MS were included. McDonald Criteria was used with the version revised in $2010^{14}$. Patients who are positive for neuromyelitis optica antibody or acute viral markers like lgM for herpes simplex virus were excluded. Patients who have a history of autoimmune diseases like systemic lupus enthematosus were also excluded. 


\section{Radiographic Features}

Two neurosurgeons (MSK, HJ) evaluated the MRI images on a picture archiving and communication system workstation monitor concerning longitudinal and axial locations, size of the lesion on T2-W, portion and pattern of enhancement, fusiform dilatation, and the presence of syringomyelia, tumoral cyst, or hemorrhage.

The lesion's longitudinal location was assessed by the dominant portion of involvement among the cervical, thoracic, and lumbar regions. Axial location was classified as the involvement of a single half or both halves of the spinal cord. The size of the lesion was measured in the number of vertebral segments involved in T2-W. The enhancing portion was classified as non-enhanced, focal, and diffuse. It was evaluated as diffuse if the lesion's enhancing component was $50 \%$ larger than the entire high intensity on T2-WM, focal if smaller than $50 \%$, or none. The pattern of contrast enhancement was classified into four patterns: solid, if the contour of the enhancing component is clear: patchy, if the contour is vague, or unclear: the rim, if only the marginal area is enhanced: or punctate if the enhancing pattern was dot-like. Fusiform dilatation was determined by whether the thickness of the cord was significantly increased compared with the peripheral area in the sagittal plane. Syringomyelia was defined as cystic dilatation of the central portion of the spinal cord without any enhancement. If a cyst was seen in the solid portion of the lesion, whether it appears centrally or peripherally, it was regarded as a tumoural cyst. A rim of extreme low signal intensity was seen at the tumor's proximal or distal pole on T2-W, suggesting haemosiderin deposits; it was defined as hemorrhage.

\section{Demographic and Clinical Features}

Demographic (sex and age) and clinical information were collected for all participants from patient records. Pathological findings in patients who underwent surgery were also reviewed. The clinical course and final diagnosis for each participant were investigated. Clinical features of neurological deficits (motor, sensory, pain, autonomic, visual symptoms) were also investigated.

\section{Statistical Analysis}

All analyses were performed with SPSS version 20.0 (IBM Corp., Armonk, NY, USA). Demographic data of SIA and SMS groups were assessed by Fisher's exact test, student $t$-test, and the preoperative results and clinical data were assessed by the Mann-Whitney test. All p-values less than 0.05 were considered statistically significant.

\section{RESULTS}

\section{Clinical Features}

In SIA, 13 lesions were identified in 13 patients, and SMS was recognized in 31 lesions in 25 patients.

SIA group had seven low-grade and six high-grade astrocytomas.
Table 1. Comparison of the clinical factors of the groups

\begin{tabular}{lccl}
\hline \hline \multicolumn{1}{c}{ Variables } & $\begin{array}{c}\text { Spinal intramedullary } \\
\text { astrocytoma }\end{array}$ & $\begin{array}{c}\text { Spinal multiple } \\
\text { sclerosis }\end{array}$ & p-value \\
\hline Patients & 13 & 25 & \\
Lesions & 13 & 31 & \\
Age (years) & $34.9 \pm 15.7$ & $42.1 \pm 15.4$ & 0.183 \\
Sex (male:female) & $8: 5$ & $8: 17$ & 0.098 \\
Symptom duration & $4.9 \pm 4.4$ & $2.1 \pm 3.6$ & $0.008^{*}$ \\
to visit (months) & & & \\
Clinical course & $\begin{array}{c}\text { Progressive, } \\
\text { non-remitting 100\% }\end{array}$ & $\begin{array}{c}\text { Non-remitting } \\
3.2 \%\end{array}$ & $<0.001^{*}$ \\
\hline
\end{tabular}

The data is presented as mean \pm standard deviation or number.

*The difference between the values of both groups was statistically significant.

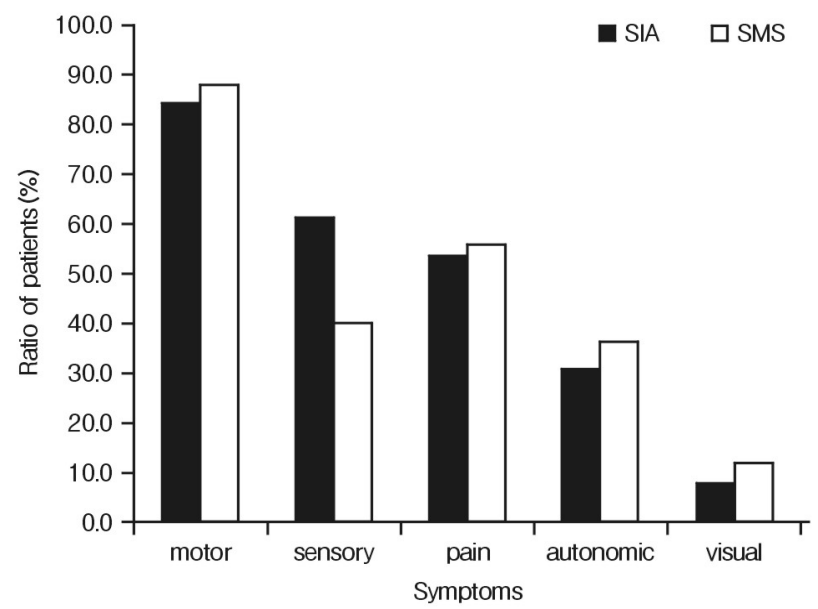

Fig. 1. Comparing spinal intramedullary astrocytoma (SIA) and spinal multiple sclerosis (SMS) symptoms, there was no significant difference in symptom ratios between the 2 groups.

Table 1 compares the clinical features. The mean age was $34.9 \pm$ 15.7 years in SIA patients and $42.1 \pm 15.4$ years in SMS patients. There was no significant difference in sex ratio. Compared with the time interval from the onset of the symptoms to visit the clinic, the SIA group showed a significantly longer duration $(4.9 \pm 4.4$ vs. $2.1 \pm 3.6$ months, $p=0.008)$. The clinical course also showed a significant difference between the two groups. In the SIA group, all patients showed a progressive course, whereas only one patient in the SMS group showed a primary progressive pattern $(p<0.001)$. Seven patients of the SMS group had a clinically isolated syndrome and remained clinically stable. Seventeen patients had a relapsingremitting pattern but showed partial recovery during the deterioration period. The most common symptom was a weakness of the extremities, followed by sensory change and axial pain. There was no difference in the ratio of the clinical symptoms in both groups (Fig. 1).

\section{Radiographic Features}

Table 2 compares the radiographic features. In the SIA group, 
Table 2. Comparison of the radiographic factors of the groups

\begin{tabular}{lccr}
\hline \multicolumn{1}{c}{ Variables } & Spinal intramedullary astrocytoma & Spinal multiple sclerosis & p-value \\
\hline Location (cervical : thoracic : conus) & $8: 4: 1$ & $17: 12: 2$ & 0.879 \\
Involved segment (vertebral body) & $3.0 \pm 1.4$ & $3.0 \pm 1.9$ & 0.461 \\
Infiltration of both halves of the spinal cord in axial plane & $13(100.0 \%)$ & $12(38.7 \%)$ & $<0.001$ \\
Fusiform dilatation & $13(100.0 \%)$ & $14(45.2 \%)$ & $<0.001$ \\
Portion of enhancement (non : focal : diffuse) & $2: 3: 8$ & $5: 13: 6$ & 0.104 \\
Pattern of Enhancement (patchy : rim : solid : punctate) & $7: 2: 2: 2$ & $10: 3: 6: 5$ & 0.857 \\
Syringomyelia & $2(15.4 \%)$ & $1(3.2 \%)$ & 0.204 \\
Tumoural cyst & $5(38.5 \%)$ & $0(0 \%)$ & $0.001^{*}$ \\
Hemorrhage & $3(23.1 \%)$ & $1(3.2 \%)$ & 0.071 \\
\hline
\end{tabular}

*The difference between the values of both groups was statistically significant.

the incidence of infiltration of both halves of the spinal cord in the axial plane was $100 \%$, but only $40 \%$ in the SMS group had both infiltrations $(p<0.001)$. Besides, all SIA patients showed fusiform dilatation of the spinal cord in the sagittal plane, which occurred in only $45.2 \%$ of the SMS patients $(p<0.001)$. The tumoral cyst was not obsenved in persons with SMS, but it was identified in 5 cases among 13 persons with $S I A(p=0.001)$. There was no difference in the location or the length of the lesion, portion or pattern of enhancement, presence of syrinx, or hemorrhage.

\section{DISCUSSION}

SMS is an immune-mediated, demyelinating disease of the CNS, and it is usually called a tumefactive lesion because it imitates a spinal cord tumor, including SIA. Despite the development of MRI, there are no established criteria to discriminate the two disease entities preoperatively. Clinically, both diseases were reported to have similar symptoms, a nonspecific combination of neurologic deficits like quadri- or para-paresis, sensory disturbance, and autonomic symptoms ${ }^{1,5)}$.

However, there were two different clinical features in disease progression between the two groups. First, while most SMS groups showed a period of symptom resolution after a clinical attack, all SIA persons showed continuous progression. MS is divided into four categories as described below; Relapsing-remitting, primary progressive, secondary progressive, progressive-relapsing type. The relapsing-remitting type, which has a flare-up period of symptom followed by a recovery time, is most common, affecting $85 \%$ to $90 \%$ of MS ${ }^{5}$. Although the course of SMS is highly variable, $95.8 \%$ of SMS patients in this series experienced a temporary symptomatic improvement after the first attack, ${ }^{1,10,12)}$. On the contrary, SIA may have an expansile characteristic so that the spinal cord is gradually pressed to show clinical deterioration ${ }^{2)}$. Brinar et al. ${ }^{4)}$ analyzed five patients and reported a similar pattern.

Second, persons with SIA took a relatively long time to visit the hospital after symptoms. The progress of SIA is mainly known to be slow, especially in low-grade tumors. It takes a significantly longer time for the low-grade tumor to be diagnosed after the onset of symptoms than in the high-grade ${ }^{17,18)}$. Thus, even if a tumor develops, it takes a relatively long time for subsequent symptoms to occur, ${ }^{1,13)}$. While SIA gradually progresses to develop symptoms over the years", clinical features that suggest demyelination have an acute or subacute onset over days to weeks, within average four to seven weeks ${ }^{5,10)}$.

The spinal cord is a small, pulsating structure that adds technical difficulties to MRI. The low relevance of MRI findings to clinical symptoms is also a source of distress ${ }^{111}$. Moreover, there is no large-scale study on the susceptibility score because the incidence is very low compared with brain lesions. These diseases may also have a heterogeneous appearance due to clinical course or phase ${ }^{21)}$. While there appears to be no unique radiologic difference SIA and SMS, some image findings can be used as a discriminatory reference.

Involving both halves of the spinal cord in the axial plane was significantly lower in the SMS group. Eckstein commented in their differential CNS demyelination strategy from MS that SMS often involves only a partial cross-sectional area of the spinal cord with less than two vertebral bodies ${ }^{7}$. Brinar et al. ${ }^{4)}$ suggested that MRI of SMS is featured by small, well-circumscribed, asymmetrically involving, focal T2-hyperintensity lesions as a differential approach to spinal cord enlargement. Tartaglino et al. ${ }^{20)}$ analyzed 124 demyeli -nating plaques in sixty-eight patients and reported 81 percent of lesions occupied less than half of the cord's cross-sectional area. In contrast, most astrocytomas were usually located in the central portion or involved the entire cord ${ }^{6,19}$. Because astrocytoma is predominantly involved in the central and dorsal portion of the entire cord, the surgical technique description recommends a common approach through the dorsal median sulcus ${ }^{9}$.

Fusiform dilatation helps in differentiating between SMS and SIA. Spinal cord enlargement is rarely seen in non-neoplastic lesions ${ }^{8,18}$. Abd-El-Barr et al." emphasized in their review of SIA that infiltrating astrocytomas often appear expansile, hypo- to iso-intense on Tl, and hyperintense on T2. If the lesion shows mass effect or cord exp ansion, it is recommended that SIA be raised as a differential diagnosis $^{4,6,15,19)}$. In the illustrative cases, the fusiform dilatation filling the entire spinal canal was observed in persons with SIA, and the lesion involved both halves. In contrast, the SMS had four vertebral bodies but relatively more minor fusiform dilatation, and only the left eccentric location was shown in an axial plane (Fig. 2, 3). 

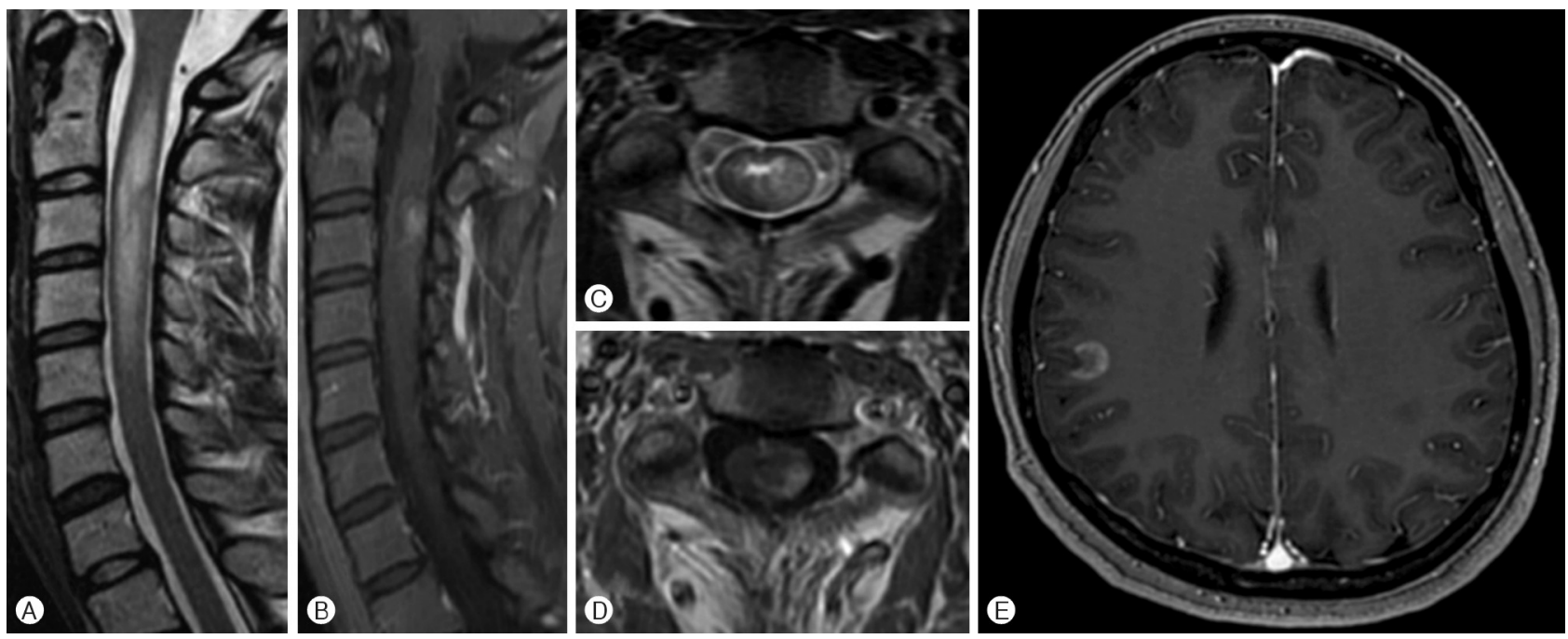

Fig. 2. An illustrative case of spinal multiple sclerosis (SMS). Magnetic resonance imaging (MRI) of a 31 -year-old female with quadriparesis for 2 months. T2-weighted image (T2-W) showed the 4-segment length of the high-intensity lesion with focal enhancement. (A, B) In the axial plane, only the left dorsal was infiltrated. (C, D) Despite conservative treatment with steroid pulse therapy for 3 months, the lesion showed progression. She underwent surgical resection of the lesion, and the pathologic report revealed multifocal perivascular lymphocytic infiltration. T2-WI in brain MRI in the postoperative period showed juxtacortical lesions (E).
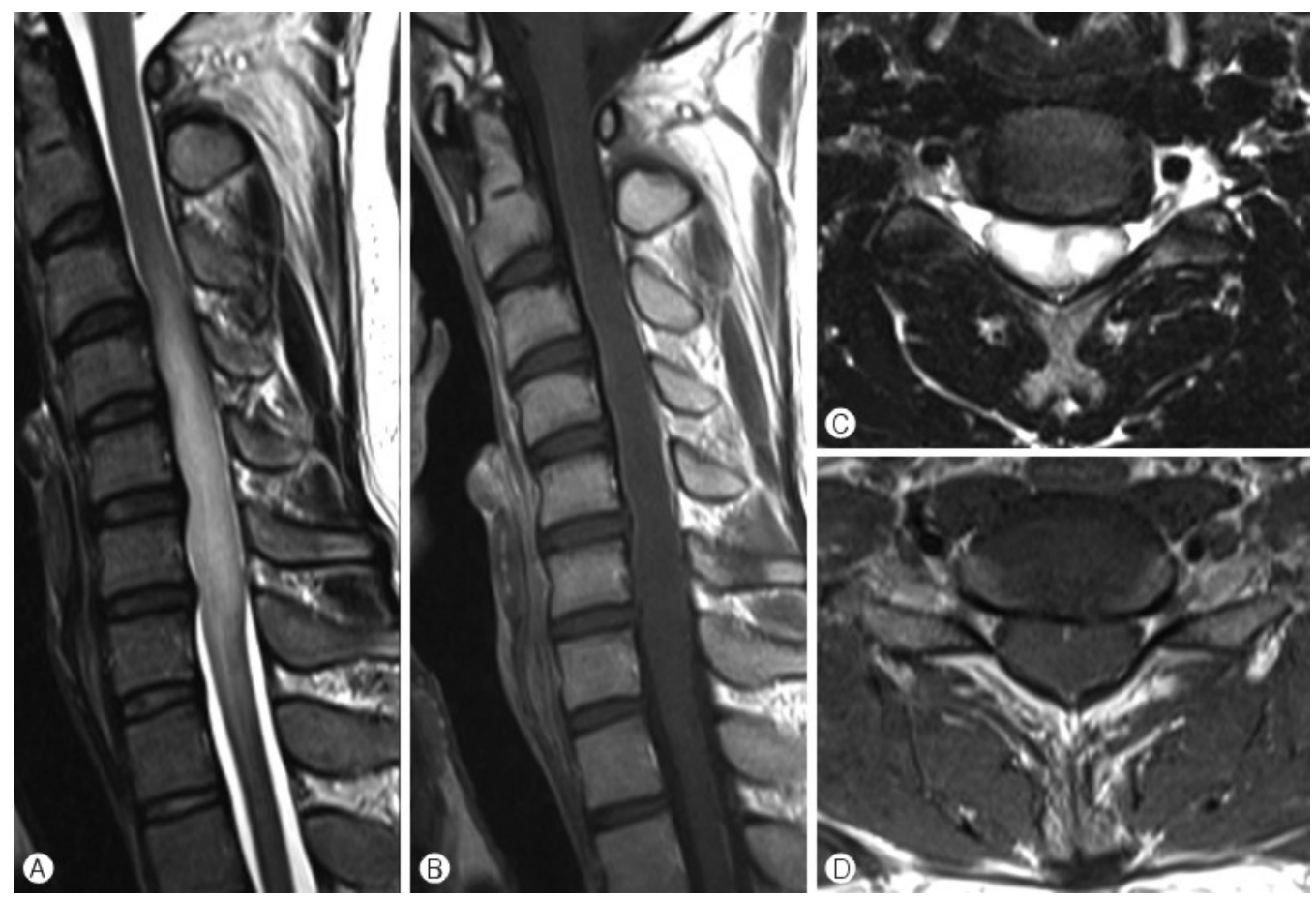

Fig. 3. An illustrative case of spinal intramedullary astrocytoma (SIA). The 23-year-old male complained of progressive both hand weakness for 9 months. In preoperative magnetic resonance imaging, the fusiform dilatation and infiltration of both halves were definite. (A, C) The contrast enhancement was not prominent. (B, D) Due to the progressive clinical course, he undenwent surgical removal of the tumor and was diagnosed with intra- medullary anaplastic astrocytoma.

Cysts are present in $25 \%$ to $40 \%$ of spinal cord neoplasms ${ }^{6,15)}$. The presence of a cyst can be interpreted as suggesting that the possibility of SMS is low. In particular, astrocytomas demonstrate tumor cysts more frequently than other intramedullary tumors ${ }^{16)}$. 
In our study, no cyst was observed in the SMS group, consistent with previous literature. However, a comprehensive judgment is required because there is a rare case report that cyst is accompanied by $\mathrm{SMS}^{3}$, and it may appear indistinguishable from solid tumors on T2-Wl as a consequence of the high protein content of the cyst fluid.

Contrast-enhanced images help the differential diagnosis ${ }^{15,20)}$, but this study did not show any difference between the two groups. Syringomyelia or hemornhage also did not show significant differences. Based on the above two clinical and three radiologic characteristics, the differential diagnosis prediction model showed high sensitivity and specificity. While it is difficult to accept the accuracy of the prediction model because of a relatively small number of subjects, it is reasonable to give meaning to the clinical and radiographic factors mentioned above.

In addition to the retrospective study design, this study has several other limitations. First, this study analyzes a small number of patients, including the 13 SIAs and 25 SMS patients, and consequently, it is not free from selection bias. Second, although astrocytoma's clinical course differs between low-grade and high-grade, the incidence of spinal cord lesions is very low, so in this study, the two diseases were classified into one group. Third, this study has heterogeneity by including both 1.5 Tesla (T) and 3T MRI and advanced MRI techniques, which have higher sensitivity, such as diffusion-weighted imaging and diffusion tensor imaging were not included. In T2-W, the probability of finding a cord lesion was reduced due to pulsation artifact, and spinal cord lesions usually do not show low intensity on $\mathrm{T} 1$ images due to the spinal cord's dense organization ${ }^{1)}$. However, it is inevitable because of the rare incidence of both diseases. Nevertheless, the existing researches on this subject were sparse, so that the clinical meaning of this study can be adequately strengthened.

\section{CONCLUSION}

In this study, clinical features and imaging characteristics were compared through a series of SIA and SMS patients. Whereas SMS patients had a remission period between acute exacerbations, whereas SIA patients showed a progressive course. The majority of the SIA occupied both halves of the spinal cord. Fusiform dilation or tumoral cyst was a common finding in SIA. However, additional studies based on large patients and advanced imaging are needed to validate these findings.

\section{CONFLICTS OF INTEREST}

No potential conflict of interest relevant to this article was reported.

\section{REFERENCES}

1. Abd-El-Barr MM, Huang KT, Chi JH: Infiltrating spinal cord astrocytomas: Epidemiology, diagnosis, treatments and future directions. J Clin Neurosci 29:15-20, 2016

2. Bowers DC, Weprin BE: Intramedullary spinal cord tumors.
Curr Treat Options Neurol 5:207-212, 2003

3. Braverman DL, Lachmann EA, Tunkel R, Nagler W: Multiple sclerosis presenting as a spinal cord tumor. Arch Phys Med Rehabil 78:1274-1276, 1997

4. Brinar M, Rados M, Habek M, Poser CM: Enlargement of the spinal cord: Inflammation or neoplasm? Clin Neurol Neurosurg 108:284-289, 2006

5. Brownlee WJ, Hardy TA, Fazekas F, Miller DH: Diagnosis of multiple sclerosis: Progress and challenges. Lancet 389:13361346, 2017

6. Do-Dai DD, Brooks MK, Goldkamp A, Erbay S, Bhadelia RA: Magnetic resonance imaging of intramedullary spinal cord lesions: a pictorial review. Curr Probl Diagn Radiol 39:160185, 2010

7. Eckstein C, Saidha S, Levy M: A differential diagnosis of central nervous system demyelination: beyond multiple sclerosis. J Neurol 259:801-816, 2012

8. Hardy TA, Chataway J: Tumefactive demyelination: an approach to diagnosis and management. J Neurol Neurosurg Psychiatry 84:1047-1053, 2013

9. Jallo GI, Kothbauer KF, Epstein FJ: Intrinsic spinal cord tumor resection. Neurosurgery 49:1124-1128, 2001

10. Lucchinetti CF, Gavrilova RH, Metz I, Parisi JE, Scheithauer BW, Weigand S, et al.: Clinical and radiographic spectrum of pathologically confirmed tumefactive multiple sclerosis. Brain 131:1759-1775, 2008

11. Lycklama G, Thompson A, Filippi M, Miller D, Polman C, Fazekas F, et al.: Spinal-cord MRI in multiple sclerosis. Lancet Neurol 2:555-562, 2003

12. Makary MS, Kirsch CF: Tumefactive demyelinating disease with isolated spinal cord involvement. Acta Radiol Short Rep 3:2047981614539324, 2014

13. Mishima K, Nakamura M, Nakamura H, Nakamura O, Funata $\mathrm{N}$, Shitara N: Leptomeningeal dissemination of cerebellar pilocytic astrocytoma. Case report. J Neurosurg 77:788-791, 1992

14. Polman CH, Reingold SC, Banwell B, Clanet M, Cohen JA, Filippi M, et al.: Diagnostic criteria for multiple sclerosis: 2010 revisions to the McDonald criteria. Ann Neurol 69:292302, 2011

15. Rogers SR, Phalke VV, Anderson J, Riccelli LP, Gonda S, Pollock JM: HEALSME: Differential diagnosis for intramedullary spinal cord lesions. Neurographics 2:13-26, 2012

16. Roonprapunt C, Houten JK: Spinal cord astrocytomas: presentation, management, and outcome. Neurosurg Clin N Am 17: 29-36, 2006

17. Ryu SJ, Kim JY, Kim KH, Park JY, Kuh SU, Chin DK, et al.: A retrospective observational study on the treatment outcomes of 26 patients with spinal cord astrocytoma including two cases of malignant transformation. Eur Spine J 25:40674079, 2016

18. Schwartz TH, McCormick PC: Non-neoplastic intramedullary pathology. Diagnostic dilemma: to Bx or not to Bx. J Neurooncol 47:283-292, 2000

19. Seo HS, Kim JH, Lee DH, Lee YH, Suh SI, Kim SY, et al.: Nonenhancing intramedullary astrocytomas and other MR imaging features: a retrospective study and systematic review. 
AJNR Am J Neuroradiol 31:498-503, 2010

20. Tartaglino LM, Friedman DP, Flanders AE, Lublin FD, Knobler RL, Liem M: Multiple sclerosis in the spinal cord: MR appearance and correlation with clinical parameters. Radiology 195:725-732, 1995
21. Thorpe JW, Kidd D, Moseley IF, Kenndall BE, Thompson AJ, MacManus DG, et al.: Serial gadolinium-enhanced MRI of the brain and spinal cord in early relapsing-remitting multiple sclerosis. Neurology 46:373-378, 1996 